\title{
Avaliação Genética de Bovinos da Raça Holandesa Usando a Produção de Leite no Dia do Controle ${ }^{1}$
}

\section{William José Ferreira 2 , Nilson Milagres Teixeira ${ }^{3}$, Ricardo Frederico Euclydes ${ }^{4}$, Rui da Silva Verneque ${ }^{3}$, Paulo Sávio Lopes ${ }^{4}$, Robledo de Almeida Torres ${ }^{4}$, Amauri Arias Wenceslau ${ }^{2}$, Marcos Vinícius Gualberto Barbosa da Silva ${ }^{2}$, Márcio Nery Magalhães Júnior ${ }^{5}$}

\begin{abstract}
RESUMO -Utilizaram-se 153.963 controles mensais de produção de leite e 13.273 primeiras lactações de vacas da raça Holandesa, com partos entre 1989 a 1998, com o objetivo de estimar parâmetros genéticos, fenotípicos e de meio ambiente para produção de leite no dia do controle (PLDC) e produção até 305 dias de lactação (P305), bem como verificar a conveniência de se utilizar a PLDC em avaliações genéticas, em substituição à P305. Foram utilizados quatro modelos (modelo animal). Em dois, modelos 1 e 2, consideraramse os controles mensais de produção, coletados ao longo da lactação, como medidas repetidas de um animal, diferenciados segundo o critério de formação de grupos de animais contemporâneos. No modelo 1 (PLDCM01), as produções dos animais foram agrupadas segundo o rebanho-ano-estação de controle de produção, enquanto, no modelo 2 (PLDCM02), o agrupamento considerou o rebanho-ano-estação de parto. No modelo 3, analisaram-se os controles mensais de produção, como características individuais (C01 até C10); e no modelo 4, analisou-se a tradicional P305. As análises foram realizadas utilizando-se o método da Máxima Verossimilhança Restrita, por meio do sistema MTDFREML. As estimativas de herdabilidade para PLDC, com o uso do modelo 1, modelo 2 e para P305, foram de $0,27,0,15$ e 0,25 , respectivamente. As herdabilidades para os controles mensais variaram de $0,11 \pm 0,02$ (C01) a 0,21 $\pm 0,03$ (C08), e os maiores valores ocorreram a partir do quarto mês. A correlação de ordem entre os valores genéticos obtidos, para P305 e para PLDC (modelo 1), foi de 0,62, para touros, e de 0,78, para vacas. Concluiu-se que é viável a utilização da PLDC em estudos envolvendo a produção de leite e que os controles do meio da lactação, se usados para seleção, podem apresentar vantagens em relação à P305.
\end{abstract}

Palavras-chave: controle mensal, método REML, modelo animal, produção de leite, raça Holandesa

\section{Genetic Evaluation of Holstein Cattle Using Test Day Milk Yield}

ABSTRACT - 153,963 test day milk yield records and 13,273 first lactations of Holstein cows calving between 1989 and 1998 , were used with the objective of estimating genetic, phenotypic and environmental parameters for test day milk yield (PLDC) and 305 day milk yield (P305) and to study the convenience of using test day yields in genetic evaluations to replace P305.Four models were used. Models 1 and 2 differed according contemporary grouping and monthly milk records were considered as repeated measures. In model 1 (PLDCM01) records were grouped by herd-year-season of test day yield and in model 2 (PLDCM02) by herd-year-season of calving. In a third (model 3), monthly yield records were analyzed as individual traits (C01 to C10); and the fourth (model 4) was the traditional 305-day model. Restricted Maximum Likelihood methodology was used with the MTDFREML system. The estimates of heritability for PLDC, using model 1 , model 2 and for P305 were $0.27,0.15$ and 0.25 , respectively. Heritabilities for monthly milk records ranged from $0.11 \pm 0.02(\mathrm{C} 01)$ to $0.21 \pm 0.03$ (C08), with the largest values occurring beginning in the fourth month. Genetic correlation estimates between monthly records and P305 ranged from 0.76 to 1.00 , with the highest correlations occurring in the middle lactation.It was concluded that using test day milk yield is promising and that selecting for middle lactation records could have advantages over 305-day milk yield.

Key Words: animal model, Holstein, milk yield, REML methodology, test day yields

\section{Introdução}

A medida padrão de produção de leite mais usada nas avaliações genéticas de vacas e touros é a produção até 305 dias de lactação (P305). A produção de cada mês, calculada com base na produção de leite no dia do controle (PLDC), é acumulada com o total de meses anteriores, para estimação da produção até 305 dias. Mesmo em países de pecuária leiteira mais tecnificada, até recentemente, uma vez estimada esta produção, os controles mensais eram descartados, em virtude da falta de recursos

\footnotetext{
1 Parte da tese de Mestrado em Zootecnia, do primeiro autor, na UFV-Universidade Federal de Viçosa.

${ }^{2}$ Estudante de Doutorado da UFV e Professor da Faculdade do Noroeste de Minas. Rua José Pereira Guimarães, no 43-A, centro,

Paracatu-MG, CEP: 38600-000. E-mail: ferreirawj@bol.com.br

${ }^{3}$ Pesquisador da Embrapa Gado de Leite e Bolsista do CNPq.

${ }^{4}$ Professor do Departamento de Zootecnia da UFV.

5 Técnico da ACGHMG-Associação de Criadores de Gado Holandês de Minas Gerais.
} 
computacionais para armazenamento (Ptak \& Schaeffer, 1993; Wiggans \& Goddard, 1997). Com os recentes avanços na área de informática, este descarte não é mais necessário. Os controles podem, então, ser usados em estudos da curva de lactação de fatores que afetam cada controle e para avaliações genéticas dos animais.

Atualmente, nas avaliações genéticas em gado de leite, modelos de repetibilidade, em que a PLDC é analisada como medida repetida, têm sido usados em substituição à modelos para P305 (Swalve, 1995a,b; Schaeffer et al., 2000). Países como os Estados Unidos, Canadá, Austrália, Nova Zelândia, entre outros, já utilizam modelos para a PLDC, em avaliações de características produtivas e contagem de células somáticas (Meyer et al., 1989; Reents et. al., 1995; Swalve, 2000). Têm-se observado muitas vantagens de tal mudança. As herdabilidades estimadas para a PLDC têm sido similares às encontradas para P305 (Meyer et al., 1989; Danell, 1982; Ptak \& Schaeffer, 1993). Além disso, os modelos para PLDC podem considerar a forma da curva de lactação, o efeito do número de dias em produção, o efeito peculiar do dia do controle para todas as vacas e os efeitos específicos para cada vaca no dia do controle, tais como número de ordenhas, duração do período seco, duração do período de serviço anterior e corrente, prenhez ou doença. As vacas podem ser avaliadas antes que suas lactações sejam encerradas, sem necessidade da utilização de fatores de ajustamento ou projeção da produção. Também, os touros podem ser avaliados com maior confiabilidade, uma vez que poderá haver maior número de filhas com produção (Ptak \& Schaeffer, 1993; Swalve, 1995b). Com a PLDC é possível avaliar os animais mais cedo, permitindo a seleção dos melhores para a reprodução no início da vida útil, possibilitando redução no intervalo de gerações.

O uso da PLDC permite também flexibilidade nos esquemas de controle leiteiro, possibilitando redução do número de controles por lactação (Swalve, 2000). Para tanto, são necessários estudos sobre a utilização de menor número de controles nas avaliações genéticas em gado de leite.

O agrupamento rebanho-ano-estação de parto (RAE) é muito usado em avaliações genéticas em gado de leite para P305, para comparar as produções de animais sujeitos aos mesmos efeitos de meio ambiente. Nos estudos sobre a PLDC, o agrupamento poderá ser feito também, considerando a estação em que o controle de produção de leite é realizado, em vez da estação de parto. A substituição de RAE por rebanho-ano-estação de controle (RDC), em modelos para PLDC, possibilita a comparação de animais que têm produção controlada no mesmo período, enfatizando os efeitos de ambiente na data de controle (Swalve, 1995b). Além disso, na estimação de componentes de variância, têm-se observado redução da variância residual, aumento da variância genética e conseqüente aumento da herdabilidade para produção de leite, quando classes de RDC são usadas nos modelos para PLDC.

Assim, objetivou-se com este trabalho definir um critério de formação de grupos de animais contemporâneos em modelo de repetibilidade para PLDC, e estimar parâmetros genéticos, fenotípicos e de meio ambiente para PLDC e P305, e ainda verificar a conveniência de se utilizar a PLDC em avaliações genéticas, em substituição à P305.

\section{Material e Métodos}

Foram analisados dados de produção de leite no dia do controle, coletados ao longo da lactação, como medida repetida de um mesmo animal e como características individuais e, também, a produção até 305 dias de lactação. Os dados usados nas análises foram provenientes do Serviço de Controle Leiteiro da Associação de Criadores de Gado Holandês de Minas Gerais.

As lactações nas quais ocorreram doenças, abortos, mortes ou vendas de vacas, as lactações em andamento e também aquelas encerradas antes de 305 dias sem registro da data ou causa de secagem, não foram usadas. Também foram eliminados controles inferiores a $3 \mathrm{~kg}$ ou superiores a $45 \mathrm{~kg}$ e vacas com idade ao parto menor que 20 ou maior que 48 meses. Após as eliminações, foram calculadas as produções até 305 dias de lactação ajustadas para duas ordenhas, conforme Teixeira (1998) e as produções no primeiro e no último controle, de acordo com o método oficial regulamentado pelo Ministério da Agricultura e do Abastecimento (Brasil, 1986). Não foram calculadas as produções até 305 dias de lactação de vacas com menos de três controles mensais de produção ou com intervalo de controles maior que 75 dias, antes de completar 305 dias de lactação. Foram ainda descartadas as produções de leite até 305 dias de lactação inferiores a $1.200 \mathrm{~kg}$ ou superiores a $13.000 \mathrm{~kg}$. 
Após estas restrições, restaram 153.963 controles mensais de produção e 13.273 primeiras lactações de vacas com partos registrados no período de 1989 a 1998.

Os controles mensais de produção de leite foram divididos em intervalos de, aproximadamente, 30 dias. Uma vez que o controle leiteiro nem sempre é realizado em intervalos regulares e com o intuito de evitar eliminação dos mesmos, considerou-se um período de, no máximo, 15 dias de atraso nas realizações das pesagens mensais. Assim, como primeiro controle, foram consideradas as pesagens efetuadas no período de quatro a 45 dias após o parto; o segundo, de 30 a 75; o terceiro, de 60 a 105; e assim sucessivamente. Quando havia mais de um controle no mesmo intervalo, um deles era transferido para o intervalo seguinte, desde que este estivesse dentro dos limites do próximo intervalo; caso contrário, um deles era eliminado aleatoriamente.

Foram utilizados quatro modelos nas análises. $\mathrm{O}$ modelo 1 (PLDCM01), para observações repetidas, também usado por Ptak \& Schaeffer (1993), foi:

$$
\mathrm{y}_{\mathrm{ijkl}}=\mathrm{RDC}_{\mathrm{i}}+\mathrm{CG}_{\mathrm{j}}+\sum_{\mathrm{n}=1}^{5} \mathrm{~b}_{\mathrm{n}} \mathrm{X}_{\mathrm{n}_{\mathrm{j} k \mathrm{kl}}}+\mathrm{a}_{\mathrm{k}}+\mathrm{pe}_{\mathrm{k}}+\mathrm{e}_{\mathrm{ijkl}}
$$

em que $y_{i j k l}=$ produção de leite no dia do controle; $\mathrm{RDC}_{\mathrm{i}}=$ efeito do rebanho-ano-estação $\mathbf{i}$ em que o controle foi realizado (estações: águas, de outubro a março e seca, de abril a setembro); $\mathrm{CG}_{\mathrm{j}}=$ efeito da composição genética $\mathbf{j}$ (animais puros de origem e animais de composição genética igual ou superior a $31 / 32$ Holandês); $b_{1}=$ coeficiente de regressão linear da característica $\mathrm{y}_{\mathrm{ijkl}}$, sobre a idade da vaca no parto; $b_{2} a b_{5}=$ coeficientes de regressão da característica $\mathrm{y}_{\mathrm{ijk}}$, sobre o número de dias em lactação (DIM); $\mathrm{X}_{1}=$ idade da vaca no parto, em dias; $\mathrm{X}_{2}=\mathrm{DIM} / \mathrm{c}$, em que $c=305$ é uma constante positiva utilizada na redução da magnitude das covariâncias; $\mathrm{X}_{3}=(\mathrm{DIM} / \mathrm{c})^{2} ; \mathrm{X}_{4}=$ $\ln (\mathrm{c} / \mathrm{DIM}) ; \mathrm{X}_{5}=(\ln (\mathrm{c} / \mathrm{DIM}))^{2} ; \mathrm{ak}=$ efeito aleatório do animal $\mathbf{k} ; \mathrm{pe}_{\mathrm{k}}=$ efeito aleatório de ambiente permanente sobre a vaca $\mathbf{k}$, durante a lactação; $\mathrm{e}_{\mathrm{ijkl}}=$ erro aleatório associado a cada observação.

Admitiu-se que o efeito de ambiente permanente e o efeito do erro aleatório são independentemente e identicamente distribuídos, com média zero e variância $\sigma_{\mathrm{pe}}^{2} \mathrm{e} \sigma_{\mathrm{e}}^{2}$, respectivamente, e não-correlacionados com o efeito de animal.

Nesta análise, foram considerados somente os dados de produção de vacas com, no mínimo, três controles coletados ao longo da lactação. Além disso, os touros deveriam ter, pelo menos, cinco filhas e cada classe de RDC deveria possuir, no mínimo, três controles de filhas de, pelo menos, dois touros.

O modelo 2 (PLDCM02) é semelhante ao modelo 1, exceto que o efeito fixo de RDC foi substituído por RAE (rebanho-ano-estação de parto). Também, as restrições para os dados foram as mesmas para o modelo 1.

O modelo 3, apresentado em seguida, foi usado nas análises dos controles mensais de produção de leite, como características individuais.

$$
y_{i j k}=\operatorname{RAE}_{i}+C_{j}+\sum_{n=1}^{2} b_{n} X_{n_{i j k l}}+a_{i j k}+e_{i j k}
$$

em que $y_{\mathrm{ijk}}=$ controle mensal de produção de leite (C01 até $\mathrm{C} 10) ; \mathrm{b}_{1}=$ coeficiente de regressão linear da característica $\mathrm{y}_{\mathrm{ijk}}$, sobre a idade da vaca no parto; $\mathrm{b}_{2}=$ coeficiente de regressão linear da característica $y_{i j k}$, sobre o intervalo do parto ao primeiro controle.

Além das restrições quanto ao número de filhas por touro e de observações por classe de RAE, descritas nos modelos 1 e 2, foram excluídos os animais sem o primeiro controle.

O modelo 4, tradicionalmente usado para analisar a produção até 305 dias de lactação, foi:

$$
y_{i j k}=R A E_{i}+C G_{j}+\sum_{n=1}^{2} b_{n} X_{1_{i j k l}}+a_{i j k}+e_{i j k}
$$

em que $\mathrm{y}_{\mathrm{ijk}}=$ produção de leite até 305 dias de lactação; $b_{1}$ e b $b_{2}=$ coeficientes de regressão linear e quadrático da característica $\mathrm{y}_{\mathrm{ijk}}$, sobre idade da vaca no parto.

Para esta análise também, os touros deveriam ter, pelo menos, cinco filhas e cada classe de RAE deveria possuir, no mínimo, três registros de produção de filhas de, pelo menos, dois touros.

Além das análises de característica única, foram feitas as de características múltiplas (análise com duas características), entre os controles mensais de produção de leite (C01 até $\mathrm{C} 10)$ e a produção até 305 dias.

Em todas as análises foi utilizado um arquivo de "pedigree", contendo identificação de animal, pai e mãe, de 46.369 indivíduos, resultando em 62.885 animais diferentes na matriz de parentesco.

Os componentes de variância e covariância, necessários à estimação dos parâmetros genéticos e à predição dos valores genéticos dos animais, para as características em estudo, foram obtidos pelo método da Máxima Verossimilhança Restrita (REML), utilizando-se o aplicativo MTDFREML (Boldman et al., 1995). 
A confiabilidade dos valores genéticos, correlação de ordem entre eles e a coincidência na seleção e descarte de animais foram usadas como critério para comparação das alternativas de avaliação genética. Análises de característica única foram utilizadas para obtenção dos valores genéticos e confiabilidades. A base de comparação foram as predições dos valores genéticos para P305. Os coeficientes de correlação de ordem de Spearman foram obtidos por meio do sistema SAS (1990).

\section{Resultados e Discussão}

As médias observadas, os desvios-padrão e os coeficientes de variação para produção de leite no dia do controle, analisados pelos modelos 1 e 2 , para controles mensais (C01 até C10) e para produção de leite até 305 dias de lactação, encontram-se na Tabela 1.

As estimativas de componentes de variância, herdabilidade e repetibilidade da produção de leite no dia de controle e da produção até 305 dias, encontram-se na Tabela 2. Para produção de leite no dia do controle, analisada pelo modelo 1, observou-se aumento na variância genética aditiva e redução na variância residual, em relação à análise realizada pelo modelo 2, proporcionando, conseqüentemente, maior herdabilidade para PLDC, quando se usou o modelo 1. A diferença entre os dois modelos está na formação de grupos de animais contemporâneos. No modelo 1, as produções dos animais são agrupadas segundo o rebanho-ano-estação de controle da produção (RDC), enquanto no modelo 2 o agrupamento considera o rebanho-ano-estação de parto (RAE).

Para produção de leite no dia do controle, analisada usando o modelo 1 (PLDCM01), a herdabilidade foi semelhante às estimadas por Swalve (1995c) e Strabel \& Szwaczkowski (1997), que foram de 0,26 e 0,27 , respectivamente. Contudo, pelo modelo 2 (PLDCM02), o valor encontrado foi menor que os relatados na literatura (Swalve, 1994, 1995a e 1995c).

A herdabilidade estimada para P305 foi similar às encontradas, no Brasil, por Almeida et al. (1997) e Zambianchi et al. (1997). Entretanto, foi inferior às estimadas, em outros países, por Pander et al. (1992) e Swalve (1995a) e, no Brasil, por Machado (1997). O valor estimado foi próximo ao obtido para produção de leite no dia do controle, analisada pelo modelo 1. Este resultado diverge do relatado no trabalho de Strabel e Szwaczkowski (1997), no qual a herdabilidade para PLDC, como observação repetida, foi superior ao valor encontrado para P305. Nos estudos de Swalve (1994, 1995a e 1995c), com um modelo de repetibilidade, os valores das estimativas de herdabilidade para P305 foram maiores do que para PLDC, o que também foi constatado neste estudo, quando o modelo 2 foi utilizado.

As correlações genéticas, fenotípicas e residuais entre os controles mensais de produção de leite e

Tabela 1 - Número de observações, médias observadas, desvios-padrão e coeficientes de variação para produção de leite no dia do controle (PLDC), analisadas pelos modelos 1 e 2, para controles mensais de produção (C01 até $\mathrm{C} 10)$ e para produção de leite até 305 dias de lactação (P305)

Table 1 - Number of observations, averages, standard deviations and coefficient of variation for test day milk yields (PLDC) using models 1 and 2, for monthly test day records (C01 to C10) and for 305 days milk yield (P305)

\begin{tabular}{lcccc}
\hline $\begin{array}{l}\text { Característica } \\
\text { Trait }\end{array}$ & $\begin{array}{c}\text { Número de observações } \\
\text { Number of observations }\end{array}$ & $\begin{array}{c}\text { Média observada (kg) } \\
\text { Average }(\mathrm{kg})\end{array}$ & $\begin{array}{c}\text { Desvio-padrão } \\
\text { Standard deviation }\end{array}$ & $\begin{array}{c}\text { Coeficiente de variação (\%) } \\
\text { Coefficient of variation }\end{array}$ \\
\hline PLDC1 & 133.485 & 19,03 & 6,09 & 32,03 \\
PLDC2 & 127.034 & 19,12 & 6,09 & 31,86 \\
C01 & 15.986 & 19,81 & 5,41 & 27,32 \\
C02 & 14.687 & 21,30 & 5,76 & 27,04 \\
C03 & 14.018 & 21,02 & 5,87 & 27,94 \\
C04 & 13.226 & 20,43 & 5,95 & 29,11 \\
C05 & 12.607 & 19,74 & 6,02 & 30,51 \\
C06 & 11.831 & 19,05 & 6,09 & 31,99 \\
C07 & 11.209 & 18,21 & 5,99 & 32,88 \\
C08 & 10.297 & 17,32 & 5,89 & 33,98 \\
C09 & 9.066 & 16,35 & 5,79 & 35,41 \\
C10 & 6.567 & 15,67 & 5,68 & 36,22 \\
P305 & 10.096 & $5.485,28$ & $1.639,47$ & 29,89 \\
\hline
\end{tabular}

\footnotetext{
${ }_{1}^{1}$ Produção de leite no dia do controle, analisada usando o modelo 1.

${ }^{2}$ Produção de leite no dia do controle, analisada usando o modelo 2.

${ }^{1}$ Test day yield analyzed using model 1.

2 Test day yield analyzed using model 2.
} 
Tabela 2 - Es timativas de componentes de variância genética aditiva $\left(\hat{\sigma}_{\mathrm{a}}^{2}\right)$, permanente de meio $\left(\hat{\sigma}_{\mathrm{pe}}^{2}\right)$, residual ( $\hat{\sigma}_{\mathrm{e}}^{2}$ ) e fenotípica $\left(\hat{\sigma}_{\mathrm{P}}^{2}\right)$ e dos coeficientes de herdabilidade $\left(\hat{\mathrm{h}}^{2}\right)$ e de repetibilidade $(\hat{r})$ para produção de leite no dia do controle, analisadas pelos modelos 1 (PLDCM01) e 2 (PLDCM02), e para produção de leite até 305 dias de lactação (P305), em análises de característica única

Table 2 - Estimates of the variance components of additive genetic effect $\left(\hat{\sigma}_{\mathrm{a}}^{2}\right)$, permanent environment $\left(\hat{\sigma}_{\mathrm{pe}}^{2}\right)$, residual $\left(\hat{\sigma}_{\mathrm{e}}^{2}\right)$ and phenotypic $\left(\hat{\sigma}_{\mathrm{P}}^{2}\right.$ ) and of the coefficients of heritability $\left(\hat{\mathrm{h}}^{2}\right)$ and repeatability ( $\hat{\mathrm{r}})$ for test day yield, using models 1 (PLDCM01) and 2 (PLDCM02), and for 305 day milk yield (P305) in single trait analyses

\begin{tabular}{lccc}
\hline $\begin{array}{l}\text { Estimativa } \\
\text { Estimate }\end{array}$ & PLDCM01 & PLDCM02 & P305 \\
\hline$\hat{\sigma}_{\mathrm{a}}^{2}$ & 6,33 & 3,14 & $336.023,03$ \\
$\hat{\sigma}_{\mathrm{pe}}^{2}$ & 8,15 & 8,04 & - \\
$\hat{\sigma}_{\mathrm{e}}^{2}$ & 8,71 & 9,79 & $993.453,62$ \\
$\hat{\sigma}_{\mathrm{P}}^{2}$ & 23,19 & 20,98 & $1.329 .476,64$ \\
$\hat{\mathrm{h}}^{2}$ & 0,27 & 0,15 & 0,25 \\
$\hat{\mathrm{r}}$ & 0,62 & 0,53 & - \\
\hline
\end{tabular}

Tabela 3 - Estimativas de correlações genéticas $\left(r_{g}\right)$, fenotípicas $\left(r_{\mathrm{p}}\right)$ e residuais $\left(r_{\mathrm{e}}\right)$ entre controles mensais de produção de leite (C01 até C10) e produção até 305 dias de lactação (P305)

Table 3 - Estimates of genetic $\left(r_{q}\right)$, phenotypic $\left(r_{p}\right)$ and residual $\left(r_{e}\right)$ correlations of monthly test yield (C01 to C10) and 305 day yield (P305)

\begin{tabular}{lccc}
\hline $\begin{array}{l}\text { Controles } \\
\text { Controls }\end{array}$ & $\mathrm{r}_{\mathrm{g}}$ & $\mathrm{r}_{\mathrm{P}}$ & $\mathrm{r}_{\mathrm{e}}$ \\
\hline C01 & 0,76 & 0,60 & 0,59 \\
C02 & 0,96 & 0,71 & 0,66 \\
C03 & 0,96 & 0,76 & 0,72 \\
C04 & 0,98 & 0,79 & 0,74 \\
C05 & 1,00 & 0,79 & 0,74 \\
C06 & 1,00 & 0,81 & 0,76 \\
C07 & 0,99 & 0,81 & 0,76 \\
C08 & 0,95 & 0,80 & 0,76 \\
C09 & 0,94 & 0,76 & 0,71 \\
C10 & 0,92 & 0,67 & 0,61 \\
\hline
\end{tabular}

Tabela 4 - Estimativas dos componentes de variância genética aditiva $\left(\hat{\sigma}_{\mathrm{a}}^{2}\right)$, residual $\left(\hat{\sigma}_{\mathrm{e}}^{2}\right)$, fenotípica $\left(\hat{\sigma}_{\mathrm{p}}^{2}\right)$ e dos coeficientes de herdabilidades $\left(\hat{\mathrm{h}}^{2}\right)$ para os controles mensais de produção de leite (C01 até C10), em análises de característica única

Table 4 - Estimates of variance components of additive genetic effect $\left(\hat{\sigma}_{\mathrm{a}}^{2}\right)$, residual $\left(\hat{\sigma}_{\mathrm{e}}^{2}\right)$, phenotypic $\left(\hat{\sigma}_{\mathrm{p}}^{2}\right)$ and coefficients of heritability $\left(\hat{\mathrm{h}}^{2}\right)$ for test day yield (C01 to C10) in single traits analyzes

\begin{tabular}{lcccc}
\hline $\begin{array}{l}\text { Controles } \\
\text { Controls }\end{array}$ & $\hat{\sigma}_{\mathrm{a}}^{2}$ & $\hat{\sigma}_{\mathrm{e}}^{2}$ & $\hat{\sigma}_{\mathrm{p}}^{2}$ & $\hat{\mathrm{h}}^{2}$ \\
\hline C01 & 2,06 & 17,14 & 19,20 & $0,11 \pm 0,02$ \\
C02 & 3,15 & 17,11 & 20,26 & $0,16 \pm 0,02$ \\
C03 & 2,78 & 16,48 & 19,25 & $0,14 \pm 0,02$ \\
C04 & 3,68 & 15,55 & 19,23 & $0,19 \pm 0,02$ \\
C05 & 3,86 & 15,47 & 19,33 & $0,20 \pm 0,03$ \\
C06 & 3,74 & 16,24 & 19,97 & $0,19 \pm 0,03$ \\
C07 & 3,52 & 15,67 & 19,18 & $0,18 \pm 0,03$ \\
C08 & 3,94 & 14,84 & 18,78 & $0,21 \pm 0,03$ \\
C09 & 3,40 & 15,24 & 18,64 & $0,18 \pm 0,03$ \\
C10 & 3,23 & 15,60 & 18,82 & $0,17 \pm 0,03$ \\
\hline
\end{tabular}

produção até 305 dias de lactação encontram-se na Tabela 3. Os valores estimados foram semelhantes aos relatados em outros trabalhos (Pander et al., 1992; Gadini et al., 1997 e Machado, 1997), sendo os maiores valores das correlações encontrados para as produções do meio da lactação, do quarto ao sétimo controle. Embora os valores das correlações, para o início e final da lactação, sejam maiores que os encontrados por Gadini et al. (1997), há indicação de que as produções, no início e no final da lactação, estejam mais sujeitas às influências de meio ambiente do que as produções dos meses do meio da lactação. Embora em menor magnitude, as correlações fenotípicas e residuais seguiram a mesma tendência.

As estimativas dos componentes de variância genética, residual e fenotípica e dos coeficientes de herdabilidade para os controles mensais de produção de leite são apresentadas na Tabela 4. As herdabilidades dos controles mensais de produção aumentaram, gradativamente, até o meio da lactação; em seguida, permaneceram constantes, com pequeno declínio no final. Esses resultados estão de acordo com a tendência, observada na maioria dos trabalhos, 
de que os maiores valores ocorrem no meio da lactação. Isso pode ser explicado pela menor variação observada nas produções desses controles, devido à influência de meio ambiente que é mais expressiva no início e no final da lactação, visto que as produções do meio da lactação são mais influenciadas pelas diferenças genéticas existentes entre as vacas. Os altos valores das estimativas de variâncias residuais, para todos os controles mensais de produção, sugerem a existência de grandes diferenças de meio ambiente entre sistemas de produção, uma vez que estes estão localizados em diversos rebanhos e regiões, no Estado de Minas Gerais.

Quando se utilizam os controles individuais do meio da lactação, poderá ocorrer redução no intervalo de gerações, uma vez que os animais poderão ser avaliados mais cedo e com isto serão possíveis maiores ganhos pela seleção.

$\mathrm{Na}$ Tabela 5, são apresentadas as médias e os respectivos desvios-padrão das confiabilidades dos valores genéticos dos touros e das vacas, para P305 e PLDC, analisada usando o modelo 1 (PLDCM01), por classes de confiabilidade. As médias de confiabilidades para PLDCM01 foram superiores às obtidas para P305, sendo as diferenças maiores para as classes mais baixas de confiabilidade. Esta vantagem, quando se utiliza a produção de leite no dia do controle, pode ser atribuída ao maior número de observações para avaliar os touros e as vacas e também, ao modelo, que ajusta com maior precisão os fatores não-genéticos (Ptak \& Schaeffer, 1993; Swalve, 1995b).

As médias e os respectivos desvios-padrão das confiabilidades dos valores genéticos dos touros e das vacas para P305, C01, C05 e C10, por classes de confiabilidade, para P305, são apresentados na Tabela 6. As médias de confiabilidades, para P305 e C05, foram próximas e ambas superiores às obtidas para $\mathrm{C} 01$ e $\mathrm{C} 10$, em todas as classes, tanto para vacas quanto para touros.

A correlação de ordem entre os valores genéticos obtidos, para produção de leite até 305 dias de lactação (modelo 4) e para produção no dia do controle (modelo 1 ), foi de 0,62 , para touros, e de 0,78 , para vacas. Esses resultados foram semelhantes aos relatados por Swalve (1995a), porém inferiores aos obtidos por Ptak \& Schaeffer (1993).

Nas Figuras 1 e 2, encontram-se, respectivamente, as porcentagens de touros e vacas em comum, selecionados para produção de leite no dia do controle (modelo 1) e para produção de leite até 305 dias de lactação, para níveis crescentes de seleção para
Tabela 5 - Médias e desvios-padrão das confiabilidades dos valores genéticos dos touros e das vacas, para produção de leite até 305 dias de lactação (P305) e no dia do controle (PLDC) por classes de confiabilidade para P305

Table 5 - Means and standard deviations of sire and cow breeding value reliabilities for 305-day yield (P305) and test day yield (PLDC) by classes of reliability for P305

\begin{tabular}{|c|c|c|c|}
\hline $\begin{array}{l}\text { Confiabilidade } \\
\text { para P305 } \\
\text { Reliability for P305 }\end{array}$ & $\begin{array}{l}\text { Número } \\
\text { Number }\end{array}$ & P305 & PLDC1 \\
\hline & & $\begin{array}{c}\text { Touros } \\
\text { Sires }\end{array}$ & \\
\hline$<0,45$ & 12 & $0,39 \pm 0,06$ & $0,63 \pm 0,06$ \\
\hline $0,45-0,49$ & 27 & $0,47 \pm 0,01$ & $0,67 \pm 0,04$ \\
\hline $0,50-0,54$ & 55 & $0,51 \pm 0,01$ & $0,70 \pm 0,05$ \\
\hline $0,55-0,59$ & 40 & $0,57 \pm 0,01$ & $0,76 \pm 0,04$ \\
\hline $0,60-0,64$ & 39 & $0,62 \pm 0,01$ & $0,80 \pm 0,04$ \\
\hline $0,65-0,69$ & 35 & $0,67 \pm 0,01$ & $0,83 \pm 0,02$ \\
\hline $0,70-0,74$ & 31 & $0,72 \pm 0,01$ & $0,86 \pm 0,02$ \\
\hline $0,75-0,79$ & 22 & $0,77 \pm 0,01$ & $0,89 \pm 0,02$ \\
\hline $0,80-0,84$ & 23 & $0,81 \pm 0,01$ & $0,92 \pm 0,01$ \\
\hline $0,85-0,89$ & 30 & $0,87 \pm 0,01$ & $0,95 \pm 0,01$ \\
\hline$\geq 0,90$ & 23 & $0,93 \pm 0,02$ & $0,97 \pm 0,01$ \\
\hline Total & 337 & $\begin{array}{l}0,66 \pm 0,15 \\
\text { Vacas } \\
\text { Cows }\end{array}$ & $0,81 \pm 0,10$ \\
\hline$<0,45$ & 236 & $0,42 \pm 0,01$ & $0,62 \pm 0,02$ \\
\hline $0,45-0,49$ & 1.300 & $0,47 \pm 0,01$ & $0,64 \pm 0,02$ \\
\hline $0,50-0,54$ & 1.427 & $0,52 \pm 0,01$ & $0,68 \pm 0,02$ \\
\hline $0,55-0,59$ & 3.243 & $0,57 \pm 0,01$ & $0,70 \pm 0,01$ \\
\hline $0,60-0,64$ & 3.416 & $0,62 \pm 0,01$ & $0,72 \pm 0,01$ \\
\hline$\geq 0,65$ & 468 & $0,66 \pm 0,01$ & $0,74 \pm 0,01$ \\
\hline Total & 10.090 & $0,57 \pm 0,06$ & $0,70 \pm 0,03$ \\
\hline
\end{tabular}

${ }_{1}^{1}$ Produção de leite no dia do controle, analisada usando o modelo. 1 Test day yield analyzed using model 1.

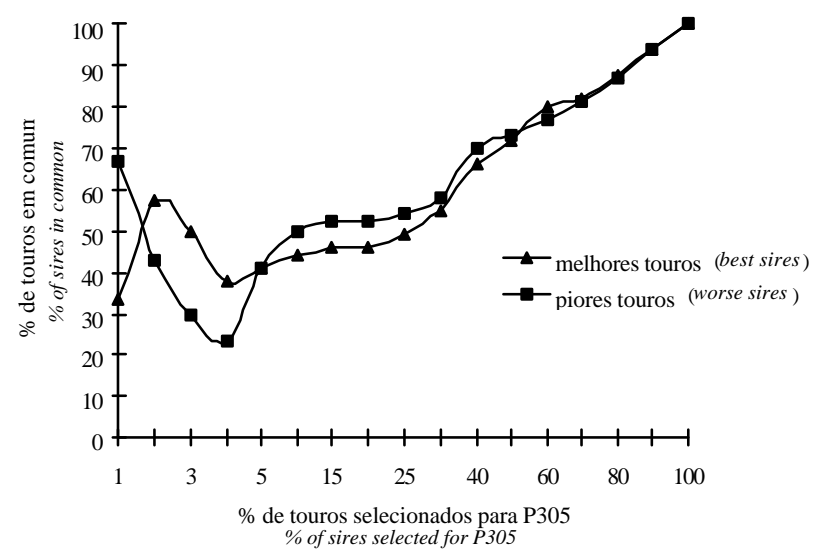

Figura 1 - Porcentagens de touros em comum, quando selecionados para PLDCM01 e P305, para porcentagens crescentes de seleção de touros para $\mathrm{P} 305$.

Figure 1 - Percentages of sires in common, when selected for PLDCM01 and P305, and increasing percentages of sires selection for $P 305$. 
Tabela 6 - Médias e desvios-padrão das confiabilidadesdos valores genéticos dos touros e das vacas, por classes de confiabilidade, para produção de leite até 305 dias de lactação (P305) e para primeiro, quinto e décimo controles mensais

Table 6 - Means and standard deviations for sire and cow breeding value reliabilities by class of reliability for 305-day yields and first fifth and tenth tests

\begin{tabular}{|c|c|c|c|c|c|}
\hline $\begin{array}{l}\text { Classe de confiabilidade } \\
\text { para } \mathrm{P} 305 \\
\text { Class of reliability } \\
\text { for } P 305 \\
\end{array}$ & $\begin{array}{l}\text { Número de } \\
\text { animais } \\
\text { Number of } \\
\text { animals } \\
\end{array}$ & $\begin{array}{l}\text { P305 } \\
\text { P305 }\end{array}$ & $\begin{array}{c}\text { Primeiro controle }(\mathrm{C} 01) \\
\text { First test }\end{array}$ & $\begin{array}{l}\text { Quinto controle }(\mathrm{C} 05) \\
\text { Fifth test }\end{array}$ & $\begin{array}{c}\text { Décimo controle }(\mathrm{C} 10) \\
\text { Tenth test }\end{array}$ \\
\hline & & & $\begin{array}{l}\text { Touros } \\
\text { Sires }\end{array}$ & & \\
\hline$<0,45$ & 4 & $0,42 \pm 0,01$ & $0,38 \pm 0,05$ & $0,45 \pm 0,05$ & $0,35 \pm 0,03$ \\
\hline $0,45-0,49$ & 9 & $0,47 \pm 0,02$ & $0,41 \pm 0,04$ & $0,47 \pm 0,04$ & $0,37 \pm 0,03$ \\
\hline $0,50-0,54$ & 23 & $0,52 \pm 0,01$ & $0,44 \pm 0,06$ & $0,51 \pm 0,05$ & $0,41 \pm 0,03$ \\
\hline $0,55-0,59$ & 33 & $0,57 \pm 0,01$ & $0,49 \pm 0,06$ & $0,57 \pm 0,04$ & $0,44 \pm 0,04$ \\
\hline $0,60-0,64$ & 36 & $0,62 \pm 0,01$ & $0,53 \pm 0,06$ & $0,61 \pm 0,04$ & $0,47 \pm 0,03$ \\
\hline $0,65-0,69$ & 33 & $0,67 \pm 0,01$ & $0,58 \pm 0,05$ & $0,66 \pm 0,03$ & $0,50 \pm 0,05$ \\
\hline $0,70-0,74$ & 30 & $0,72 \pm 0,01$ & $0,63 \pm 0,03$ & $0,71 \pm 0,02$ & $0,56 \pm 0,04$ \\
\hline $0,75-0,79$ & 22 & $0,77 \pm 0,01$ & $0,68 \pm 0,05$ & $0,76 \pm 0,03$ & $0,61 \pm 0,04$ \\
\hline $0,80-0,84$ & 23 & $0,81 \pm 0,01$ & $0,73 \pm 0,03$ & $0,80 \pm 0,02$ & $0,68 \pm 0,04$ \\
\hline $0,85-0,89$ & 30 & $0,87 \pm 0,01$ & $0,81 \pm 0,03$ & $0,86 \pm 0,02$ & $0,75 \pm 0,04$ \\
\hline$\geq 0,90$ & 23 & $0,93 \pm 0,02$ & $0,89 \pm 0,04$ & $0,92 \pm 0,02$ & $0,86 \pm 0,04$ \\
\hline Total & 266 & $0,70 \pm 0,13$ & $\begin{array}{c}0,62 \pm 0,15 \\
\text { Vacas }\end{array}$ & $0,69 \pm 0,14$ & $0,56 \pm 0,15$ \\
\hline$<0,45$ & 82 & $0,43 \pm 0,01$ & $\begin{array}{c}\text { Cows } \\
0,29 \pm 0,02\end{array}$ & $0,39 \pm 0,02$ & $0,35 \pm 0,01$ \\
\hline $0,45-0,49$ & 660 & $0,48 \pm 0,01$ & $0,32 \pm 0,02$ & $0,43 \pm 0,01$ & $0,38 \pm 0,02$ \\
\hline $0,50-0,54$ & 679 & $0,52 \pm 0,01$ & $0,39 \pm 0,03$ & $0,49 \pm 0,02$ & $0,42 \pm 0,02$ \\
\hline $0,55-0,59$ & 1.903 & $0,57 \pm 0,01$ & $0,46 \pm 0,03$ & $0,54 \pm 0,02$ & $0,48 \pm 0,03$ \\
\hline $0,60-0,64$ & 2.233 & $0,62 \pm 0,01$ & $0,53 \pm 0,02$ & $0,59 \pm 0,02$ & $0,54 \pm 0,02$ \\
\hline$\geq 0,65$ & 338 & $0,66 \pm 0,01$ & $0,57 \pm 0,01$ & $0,63 \pm 0,01$ & $0,58 \pm 0,02$ \\
\hline Total & 5.895 & $0,58 \pm 0,05$ & $0,47 \pm 0,08$ & $0,54 \pm 0,06$ & $0,50 \pm 0,06$ \\
\hline
\end{tabular}

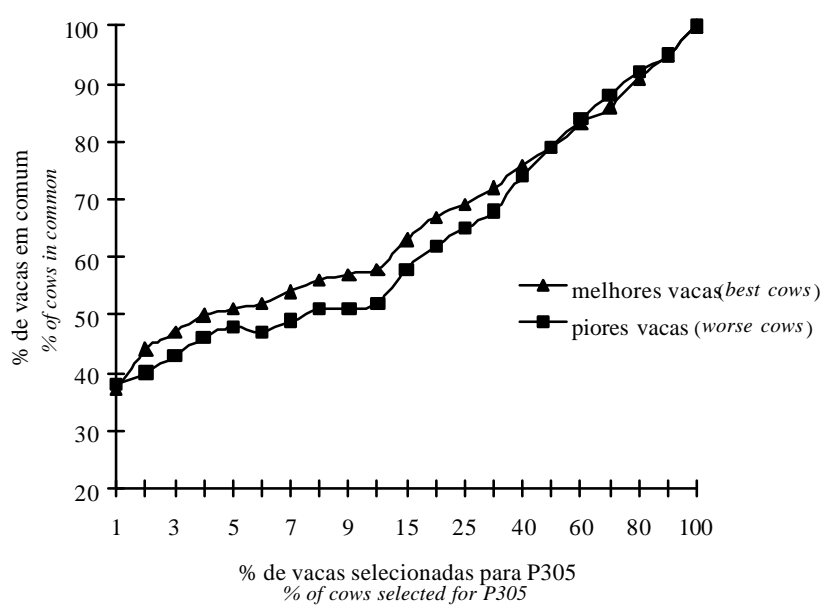

Figura 2 - Porcentagens de vacas em comum, quando selecionadas para PLDCM01 e P305, para porcentagens crescentes de seleção de vacas para P305.

Figure 2 - Percentages of cows in common, when selected for PLDCM01 and P305, and increasing percentages of cow selection for $P 305$. produção até 305 dias. Quando foram selecionados até $35 \%$, tanto de touros quanto de vacas, houve divergências entre as alternativas. A partir de então, não diferiram.

Quando foram selecionados até 30\% dos melhores touros usando-se P305, entre aqueles com confiabilidade superior a 60 ou $80 \%$ (Tabela 7), a porcentagem de touros selecionados em comum, quando se utilizaram P305 e PLDCM01 (modelo 1), foi baixa. Nas duas situações, as porcentagens de animais em comum, selecionados para P305 e PLDCM01, foram inferiores a $70 \%$, em todos os níveis de seleção.

Nas Figuras 3 e 4, encontram-se, respectivamente, as porcentagens de touros e vacas, em comum, selecionados para produção no primeiro $(\mathrm{C} 01)$, ou no quinto $(\mathrm{C} 05)$, ou no décimo $(\mathrm{C} 10)$ controles e para produção de leite até 305 dias de lactação, para níveis crescentes de seleção. As porcentagens de animais em 
Tabela 7 - Porcentagem de touros com confiabilidades superiores a 60 ou $80 \%$ em comum para P305 e PLDCM01, quando até $30 \%$ foram selecionados usando-se P305

Table 7 - Percentages of sires with reliabilities greater than 60 or $80 \%$ in common for P305 and PLDCM01 when up to $30 \%$ were selected using P305

\begin{tabular}{|c|c|c|c|c|}
\hline \multirow[t]{2}{*}{$\begin{array}{l}\text { Porcentagem selecionada } \\
\text { Selected percentage }\end{array}$} & \multicolumn{2}{|c|}{$\begin{array}{c}\text { Confiabilidade }>60 \% \\
\text { Reliability }>60 \%\end{array}$} & \multicolumn{2}{|c|}{$\begin{array}{c}\text { Confiabilidade }>80 \% \\
\text { Reliability }>80 \%\end{array}$} \\
\hline & $\begin{array}{l}\text { Número de touros } \\
\text { Number of sires }\end{array}$ & $\begin{array}{l}\% \text { de touros em comum } \\
\% \text { of sires in common }\end{array}$ & $\begin{array}{l}\text { Número de touros } \\
\text { Number of sires }\end{array}$ & $\begin{array}{l}\% \text { de touros em comum } \\
\% \text { of sires in common }\end{array}$ \\
\hline 2 & 4 & 25 & - & - \\
\hline 3 & 6 & 67 & - & - \\
\hline 4 & 8 & 63 & - & - \\
\hline 5 & 10 & 60 & 3 & 67 \\
\hline 10 & 20 & 45 & 7 & 43 \\
\hline 15 & 29 & 48 & 10 & 40 \\
\hline 20 & 39 & 51 & 14 & 43 \\
\hline 25 & 49 & 51 & 17 & 59 \\
\hline 30 & 59 & 56 & 21 & 52 \\
\hline
\end{tabular}

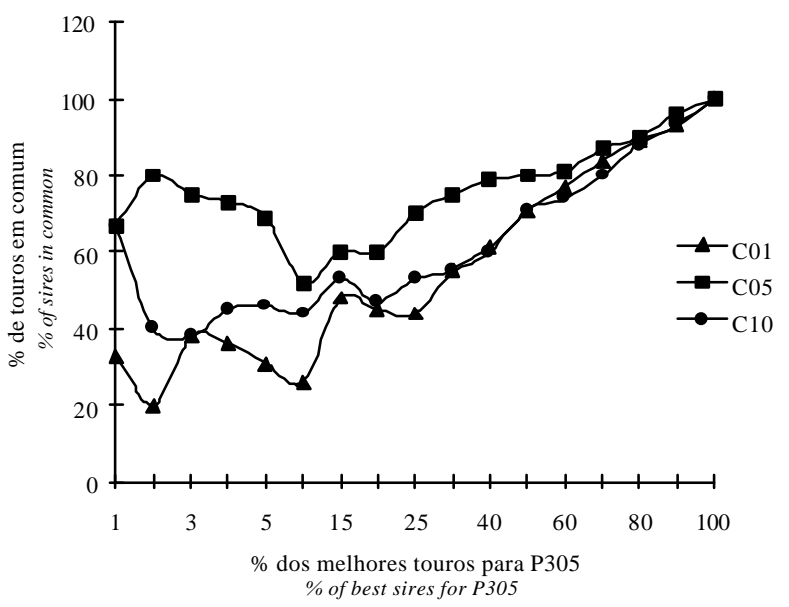

Figura 3 - Porcentagens de touros em comum, selecionados para produção no primeiro (C01), ou no quinto (C05), ou no décimo (C10) controle e para produção até 305 dias de lactação (P305), para níveis crescentes de seleção de touros.

Figure 3 - Percentages of sires in common, selected for yield in the first (C01), or fifth (C05), or tenth (C10) test and 305-day yield for increasing levels of sire selection.

comum foram maiores para C05 do que para C01 e C10. Para touros, os métodos C05 e P305 apresentaram moderado percentual de animais em comum, na maioria dos níveis de seleção para P305. Entretanto, para C01 e C10, quando foram selecionados, aproximadamente, $60 \%$ dos touros, os métodos divergiram. A mesma tendência foi observada para vacas, com maior coincidência dos métodos para C05. Contudo, para C01 e C10, não houve seleção dos mesmos animais para a maioria dos níveis de seleção para P305.

Considerando-se que é desejável menor número

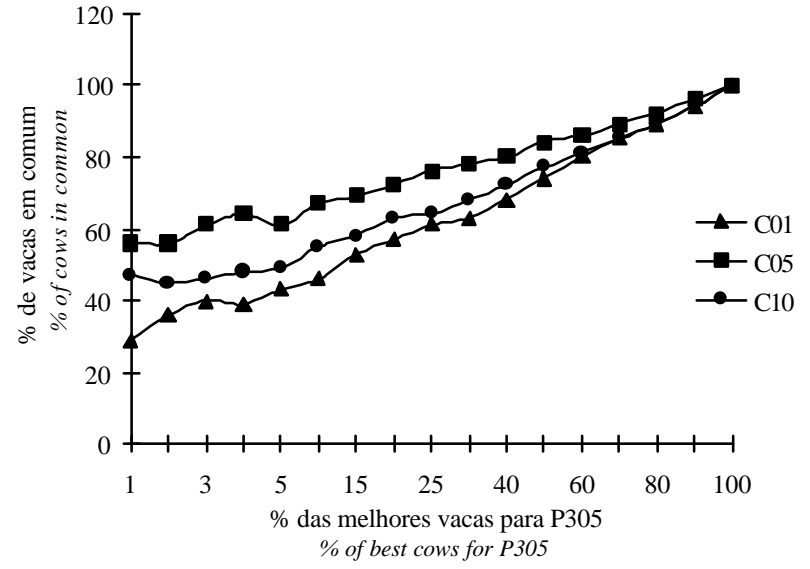

Figura 4 - Porcentagens de vacas em comum, selecionadas para produção no primeiro (C01), ou no quinto ( $\mathrm{C} 05$ ), ou no décimo (C10) controle e produção até 305 dias de lactação (P305), para níveis crescentes de seleção de vacas.

Figure 4 - Percentages of cows in common, selected for yield in the first (C01), or fifth (CO5), or tenth (C10) test and 305-day yield for increasing levels of cow selection.

de controles para redução de custos, pesquisas adicionais são necessárias para identificação dos controles a serem usados. Além disso, é necessário que se definam modelos que contemplem mais lactações da mesma vaca.

\section{Conclusões}

Em vista das vantagens de se utilizar a produção de leite no dia do controle, como observação repetida, de se constituírem grupos contemporâneos conside- 
rando-se a estação de controle leiteiro e da possibilidade de utilização dos controles mensais de produção como características individuais, seria conveniente a realização de avaliações genéticas que contemplem tais aspectos.

A produção de leite no dia do controle, em modelos de repetibilidade, quando comparada com a produção até 305 dias, possibilita aumento de confiabilidades dos valores genéticos.

As produções de leite no dia do controle, do meio da lactação, podem ser usadas para predição dos valores genéticos em substituição à produção até 305 dias, promovendo redução do número de controles e do intervalo de gerações.

\section{Agradecimento}

À Universidade Federal de Viçosa e ao Departamento de Zootecnia, pela oportunidade de realização do curso de mestrado.

À Associação de Criadores de Gado Holandês de Minas Gerais, pela concessão dos dados, essenciais à realização deste estudo.

À Coordenação de Aperfeiçoamento de Pessoal de Nível Superior (CAPES), pela concessão da bolsa de estudo.

\section{Literatura Citada}

ALMEIDA, R.; RIBAS, N.P.; MONARDES, H. Estudo dos efeitos genéticos sobre as características produtivas de vacas da raça Holandesa na região de Batavo, Paraná.In: REUNIÃO ANUAL DA SOCIEDADE BRASILEIRA DE ZOOTECNIA, 34., 1997, Juiz de Fora. Anais... Juiz de Fora: Sociedade Brasileira de Zootecnia, 1997. p.68-70.

BOLDMAN, K.G.; KRIESE, L.A.; Van VLECK, L.D. et al.A manual for use of MTDFREML. A set of programs to obtain estimates of variances and covariances. [DRAFT]. Beltsville: U.S. Department of Agriculture, Agricultural Research Service, 1995. 125p.

BRASIL. Portaria n. 45, de 10 de outubro de 1986. Normas técnicas para execução do serviço de controle leiteiro em bovídeos. Diário Oficial [da República Federativa do Brasil], Brasília, DF, n.195, p.15532-15535, 15/10/86. Seção I.

DANELL, B. Studies on lactation yield and individual test day yields of Swedish dairy cows. II. Estimates of genetic and phenotypic parameters. Acta Agriculture Scand., v.32, p.82-92, 1982.

GADINI, C.H.; KEWON, J.F.; Van VLECK, L.D. Correlações entre produções no dia do controle e em 305 dias de lactação de vacas da raça Holandesa. In: REUNIÃO ANUAL DA SOCIEDADE BRASILEIRA DEZOOTECNIA, 34., 1997, Juiz de Fora. Anais... Juiz de Fora: Sociedade Brasileira de Zootecnia, 1997. p.44-46.
MACHADO, S.G. Parâmetros genéticos e de ambiente da produção de leite no dia do controle da primeira lactação de vacas da raça Holandesa. Jaboticabal: Universidade Estadual Paulista, 1997. 75p. Dissertação (Mestrado em Zootecnia) - Universidade Estadual Paulista, 1997.

MEYER, K.; GRASER, H.U.; HAMMOND, K. Estimates of genetic parameters for first lactation test day production of Australian Black and White cows. Livestock Production Science, v.21, n.3, p.177-199, 1989.

PANDER, B.L.; HILL, W.G.; THOMPSON, R. Genetic parameters of test day records of British Holstein-Friesian heifers. Animal Production, v.55, n.1, p.11-21, 1992.

PTAK, E.; SCHAEFFER, L.R. Use of test day yields for genetic evaluation of dairy sires and cows.Livestock Production Science, v.34, n.1-2, p.23-34, 1993.

REENTS, R.; DEKKERS, J.C.M.; SCHAEFFER, L.R. Genetic evaluation for somatic cell score with a test day model for multiple lactations. Journal of Dairy Science, v.78, n. 12, p.2858-2870, 1995.

SAS INSTITUTE.SAS/STAT ${ }^{\circledR}$ user's guide,version 6.4.ed. Cary, NC: 1990, v.1, 934 p.

SCHAEFFER, L.R.; JAMROZIK, J.; KISTEMAKER, G.J. et al. Experience with a test-day model. Journal of Dairy Science, v.83, n.5, p.1135-1144, 2000.

STRABEL, T.; SZW ACZKOWSKI, T. Additive genetic and permanent environmental variance components for test day milk traits in Black-White cattle. Livestock Production Science, v.48, n.2, p.91-98, 1997.

SWALVE, H.H. Genetic relationships between testday milk production and persistency in dairy cattle performance records. In: WORLD CONGRESS ON GENETICS APPLIED TO LIVESTOCK PRODUCTION, 5., 1994, Guelph. Proceedings... Guelph: Ontário, Canadá, 1994. p.467-470.

SWALVE, H.H. The effect of test day models on the estimation of genetic parameters and breeding values for dairy yield traits. Journal of Dairy Science, v.78, n.4, p.929-938, 1995a.

SWALVE, H.H. Test day models in the analysis of dairy production data - a review.Arch. Tierz. Dummerstorf, v.38, n.6, p.591-612, 1995b.

SWALVE, H.H. Genetic relationship between dairy lactation persistency and yield. Journal of Animal Breeding Genetic, v.112, n.4, p.303-311, 1995c.

SWALVE, H.H. Theoretical basis and computational methods for different test-day genetic evaluation methods. Journal of Dairy Science, v.83, n.5, p.1115-1124, 2000.

TEIXEIRA, N.M. Padronização da produção de leite para duas ordenhas na raça Holandesa. Juiz de Fora: EMBRAPACNPGL, 1998. 1p. (EMBRAPA-CNPGL. Folha solta, 38).

ZAMBIANCHI, A.R.; FREITAS, M.A.R.; PEREIRA, C.S. Aspectos genéticos e de ambiente da produção de leite e intervalo entre partos de vacas da raça Holandesa. In: REUNIÃO ANUAL DA SOCIEDADE BRASILEIRA DE ZOOTECNIA, 34., 1997, Juiz de Fora. Anais... Juiz de Fora: Sociedade Brasileira de Zootecnia, 1997. p.13-15.

WIGGANS, G.R.; GODDARD, M.E. A computationally feasible test day model for genetic evaluation of yield traits in the United States. Journal of Dairy Science, v.80, n.8, p.1795-1800, 1997. 